\title{
Influence of wave noise on frequencies and amplitudes of the solar $p$-modes
}

\author{
K. Murawski ${ }^{1}$, L. Nocera ${ }^{2}$, and E. N. Pelinovsky ${ }^{3}$ \\ 1 Department of Complex Physical Systems, Institute of Physics, UMCS, ul. Radziszewskiego 10, 20-031 Lublin, \\ Poland \\ 2 Institute of Atomic and Molecular Physics, National Research Council, Via Moruzzi 1, 56124 Pisa, Italy \\ 3 Institute of Applied Physics, 46 Ul'anova Street 603600, Nizhny Novgorod, Russia
}

Received 16 November 2001 / Accepted 12 March 2002

\begin{abstract}
The influence of space- and time-dependent random mass density field, associated with granules, on frequencies and amplitudes of the solar $p$-modes is examined in the limit of weak random fields and small amplitude oscillations. The $p$-modes are approximated by the sound waves which propagate in the gravity-free medium. Using a perturbative method, we derive a dispersion relation which is solved for the case of wave noise for which the spectrum $E(k, \omega) \sim E(k) \delta\left(\omega-c_{\mathrm{r}} k\right)$, where $\delta$ is the Dirac's delta-function and $c_{\mathrm{r}}$ is the random phase speed. We find that at $c_{\mathrm{r}}=\omega / k$ a resonance occurs at which the cyclic frequency $\omega$ tends to infinity. For values of $c_{\mathrm{r}}$ which are close to the resonance point, the frequency shift may be both negative or positive and the imaginary part of the frequency attains the negative (positive) sign for $c_{\mathrm{r}}<\omega / k\left(c_{\mathrm{r}}>\omega / k\right)$.
\end{abstract}

Key words. convection - Sun: oscillations - turbulence

\section{Introduction}

The solar $p$-modes are essentially sound waves, for which the dominant restoring force is pressure. The $p$-modes propagate if their cyclic frequency $\omega$ is higher than the acoustic cutoff frequency $\omega_{\mathrm{ac}}=\sqrt{\gamma g / 4 h}$, where $\gamma$ is the specific heats ratio, $g$ is the gravity, and $h$ is the isothermal density scale-height. These modes satisfy the approximate dispersion relation

$\omega^{2} \simeq g k\left(1+\frac{2 n}{m}\right)$

where $k=\sqrt{l(l+1)} / R_{\mathrm{s}}$ is the horizontal wavenumber, $\omega$ is the cyclic frequency, $R_{\mathrm{s}}=696 \mathrm{Mm}$ is the solar radius, $l$ is a non-negative integer which denotes the spherical degree, $n$ is the mode number which corresponds to a number of nodes in the radial wave profile, and $m$ is an integer such that $|m| \leq l$. As the frequency grows with the radial order $n$ the spectrum of the $p$-modes is Sturmian.

A $p$-mode is trapped between an upper reflecting layer and a lower turning point. Outside this region, the $p$-mode is evanescent. The ray paths of the sound waves that do not propagate vertically are bent by the increase with

Send offprint requests to: K. Murawski, e-mail: kmurawsk@tytan.umcs.lublin.pl depth in sound speed until they reach the turning point where they undergo total internal refraction. The turning point is located the closer to the solar surface the higher is the spherical degree $l$. At the solar surface the sound waves are reflected back by the rapid decrease of mass density, where the wavelength becomes comparable to the local density scale height.

The Sun is certainly host to many dynamical phenomena, of which granulation is the most obvious, which are usually neglected in solar modeling. For the recent discussion of the effect of granulation on the solar oscillations see Nocera et al. (2001) and Murawski et al. (2001b). Granules, which exhibit random flows and consequently random mass densities (Stein \& Nordlund 1998), might therefore produce observable shifts in the frequencies of sound waves propagating across them. This effect is likely to be particularly pronounced when the waves have a wavelength that is short compared with the spatial scale of the cells. Helioseismology provides a possibility of studying the manifestation of these phenomena in the oscillation frequencies.

The paper is organized as follows. In Sect. 2 we present the dispersion relation for the random $p$-modes. In this section, we investigate the influence of the random mass densities on frequencies and amplitudes of the solar 
modes. This paper is concluded by the presentation of the main results in Sect. 3.

\section{Random $p$-modes}

The solar $p$-modes are described by the equations of hydrodynamics, with the gravity and radiative transfer terms included (Gough 1994). These general equations appear to be quite complicated even for numerical treatment. Consequently a fruitful approach to approximate these equations to a point where they can be easily discussed is proposed in this paper. It offers an insight into the behavior of the modes which are approximated locally by the sound waves (Swisdak \& Zweibel 1999; Dziembowski 2000). As a consequence of this assumption, the present analysis is performed for a uniform, not a stratified, unperturbed state. Obviously, such a state is not quite realistic and there are no velocity fluctuations. However, taking into account the realistic state is a formidable task and therefore we limit our discussion to the simpler case of the random mass density field which is driven by velocity fluctuations.

\subsection{Space- and time-dependent random mass density}

Using the standard method of random wave theory for small amplitude sound waves and weak random mass density field (e.g. Nocera et al. 2001; Murawski et al. 2001a) we derive the following dispersion relation:

$\Omega^{2}-K^{2}=\Omega^{2} \int_{-\infty}^{\infty} \int_{-\infty}^{\infty} \frac{\hat{\Omega}^{2} E(\hat{K}-K, \hat{\Omega}-\Omega)}{\hat{\Omega}^{2}-\hat{K}^{2}} \mathrm{~d} \hat{K} \mathrm{~d} \hat{\Omega}$.

Here the dimensionless wavevector $K=k l_{x}$ and the dimensionless frequency $\Omega=\omega l_{x} / c_{0}$ are introduced, $l_{x}$ is the correlation length, $c_{0}=\sqrt{\gamma p_{0} / \varrho_{0}}$ is the sound speed at equilibrium, and $E$ is the Fourier transform of the correlation function $\left\langle\varrho_{\mathrm{r}}(x, t) \varrho_{\mathrm{r}}(X, T)\right\rangle / \varrho_{0}^{2}$. Here $\varrho_{\mathrm{r}}(x, t)$ is a statistically homogeneous random density (e.g. Sobczyk 1985) with a vanishing ensemble average $\left\langle\varrho_{\mathrm{r}}\right\rangle$.

From the dispersion relation of Eq. (2) it follows that the random sound waves are no longer dispersionless. We will see in the forthcoming part of this paper that sound waves experience not only frequency shift but also amplitude alteration due to a presence of the random field.

Special limits of Eq. (2) were already considered in the literature. The case of a "frozen" (space-dependent) random field $\varrho_{\mathrm{r}}=\varrho_{\mathrm{r}}(x)$ was discussed by Nocera et al. (2001). The main conclusion was that the sound waves were attenuated by any random field. For the Gaussian statistics,

$E(K)=\frac{\sigma^{2}}{\pi} \exp \left(-K^{2}\right)$

the sound wave frequencies were lifted up and the following expression was found:

$\frac{\Omega-K}{\sigma^{2}} \simeq \frac{1}{2 \sqrt{\pi}} K^{2} D(2 K)-\frac{i}{4} K^{2}\left[1+\exp \left(-4 K^{2}\right)\right]$,

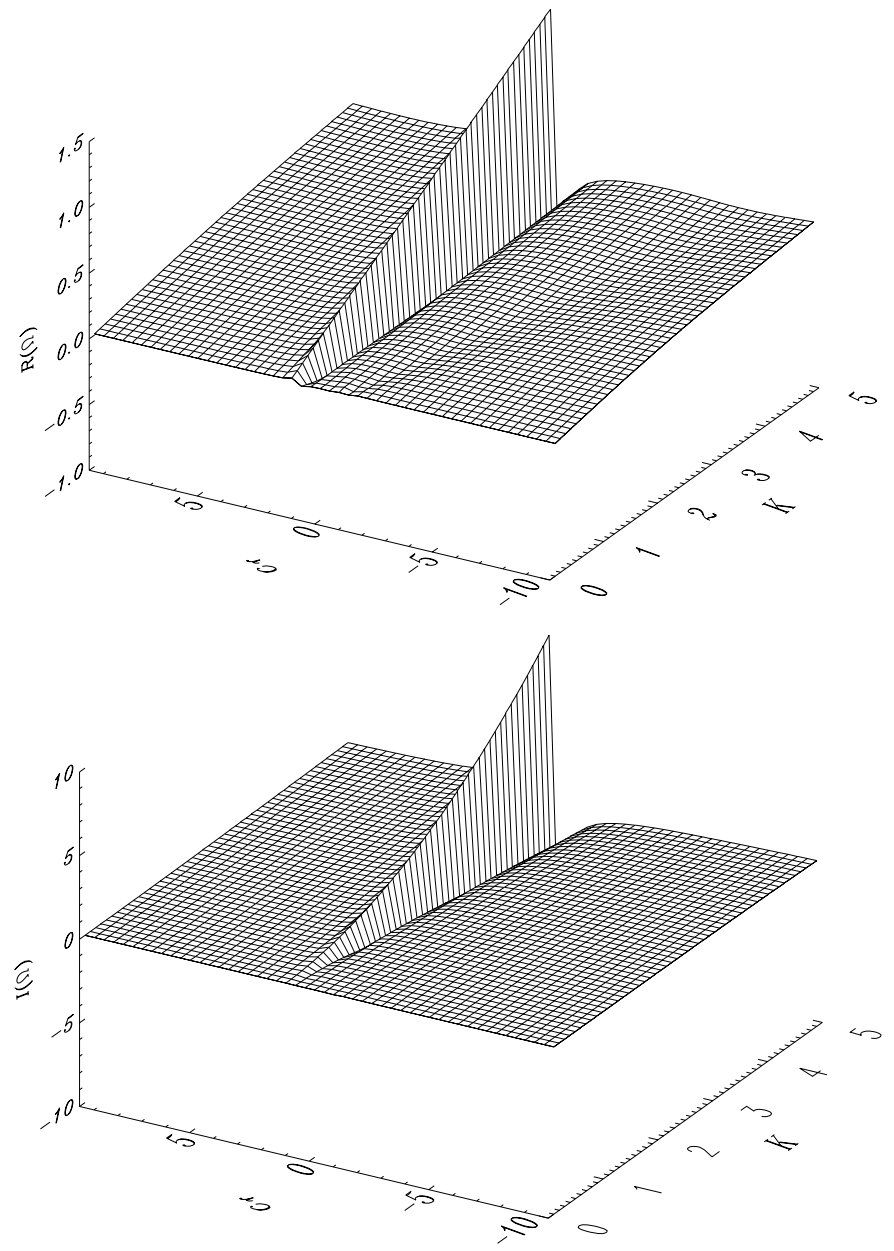

Fig. 1. Real (top panel) and imaginary (bottom panel) parts of $\Omega_{2}$.

where $D(x)=\mathrm{e}^{-x^{2}} \int_{0}^{x} \mathrm{e}^{t^{2}} \mathrm{~d} t$ is Dawson's integral (Press et al. 1992) and $\sigma^{2} \ll 1$ is a small parameter which enforces the weakness of the random density fluctuations.

The case of the time-dependent random field $\varrho_{\mathrm{r}}=\varrho_{\mathrm{r}}(t)$ was discussed by Murawski et al. (2001a) who showed that such random field leads to energy transfer from the random field to the sound waves and consequently to wave amplification. Moreover, for the Gaussian random field the frequencies of the sound waves were lifted up, similarly as in the case of the space-dependent random field.

It is natural then to inquire about the properties of the sound waves which propagate through the medium of space-and time-dependent random mass density field. The study of the doubly singular Cauchy integral associated with a fully space and time dependent random density spectrum in Eq. (2) will be left to a forthcoming paper. In the the following part of this work, we concentrate on the simpler case of wave noise.

\subsubsection{Wave noise}

We define wave noise through the spectrum

$E(K, \Omega)=\frac{\sigma^{2}}{\pi} E(K) \delta\left(\Omega-\Omega_{\mathrm{r}}(K)\right)$, 

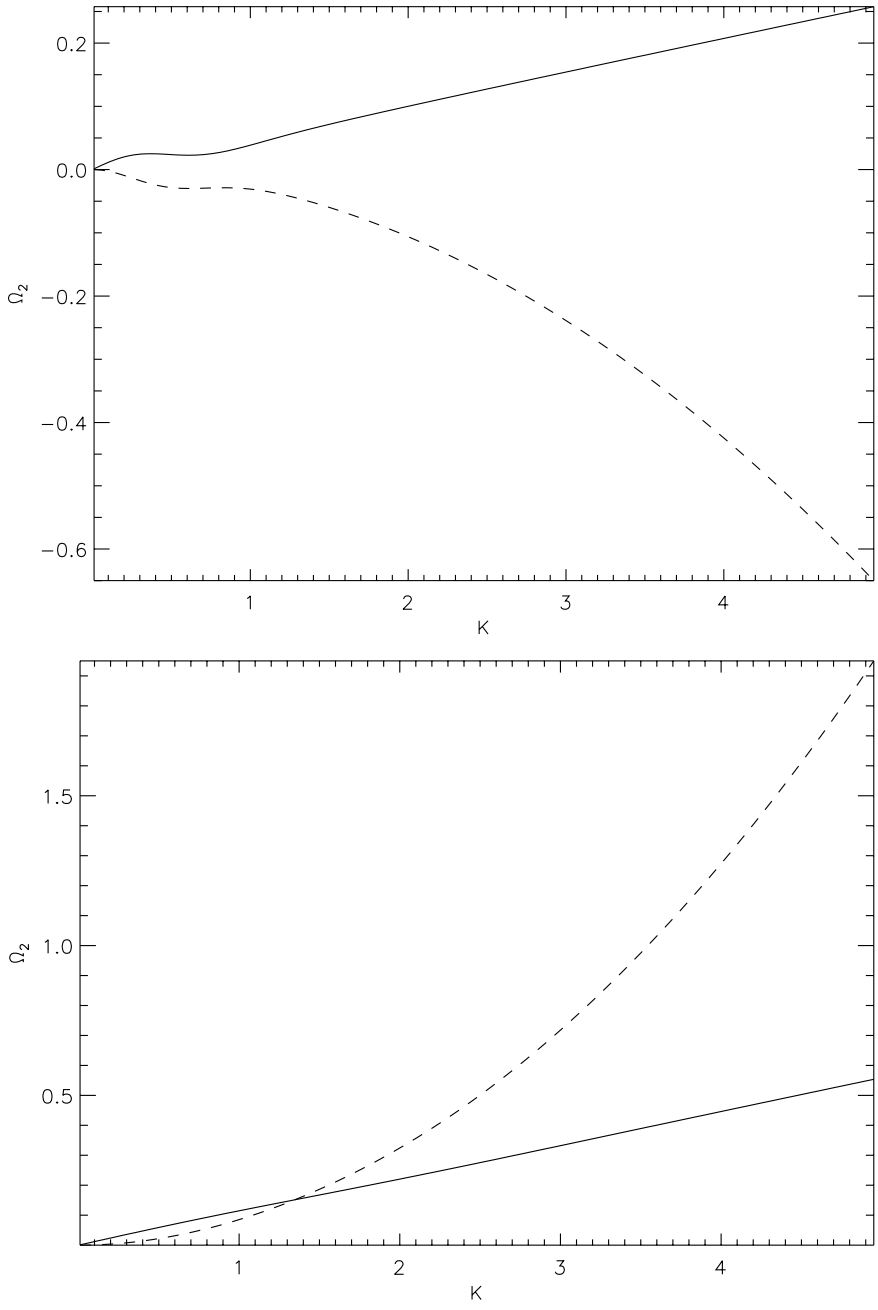

Fig. 2. Real (solid lines) and imaginary (dashed lines) parts of $\Omega_{2}$ versus $K$ for $c_{\mathrm{r}}=-2$ (top panel) and $c_{\mathrm{r}}=2$ (bottom panel).

where $\Omega_{\mathrm{r}}$ is the $K$-dependent frequency of the random density fluctuations and specialize to dispersionless noise:

$\Omega_{\mathrm{r}}(K)=c_{\mathrm{r}} K$,

where $c_{\mathrm{r}}$ is the phase speed of the random noise. For such noise the initial random mass density profile $\varrho_{\mathrm{r}}(x, t=0)$ is translated in time $t$ by the distance $c_{\mathrm{r}} t$ such that $\varrho_{\mathrm{r}}(x, t)=$ $\varrho_{\mathrm{r}}\left(x-c_{\mathrm{r}} t, t=0\right)$. For practical applications, henceforth we shall use the Gaussian spectrum of Eq. (3).

We now consider a sound wave whose wavenumber is $K$. In view of the smallness of $\sigma^{2}$, its frequency $\Omega$ given by the dispersion relation in Eq. (2), can be expanded as

$\Omega=K+\sigma^{2} \Omega_{2}+\cdots$

As in Nocera et al. (2001) we distinguish two cases: $K \neq 0$ and $K=0$. When $K \neq 0$, substituting Eqs. (5)-(7) into Eq. (2) we obtain

$\Omega_{2}=\frac{1}{2 \pi^{2}} K \int_{-\infty}^{\infty} \frac{\left(c_{\mathrm{r}} \hat{K}-c_{-} K\right)^{2} \mathrm{e}^{-(\hat{K}-K)^{2}}}{\left(c_{\mathrm{r}} \hat{K}-c_{-} K\right)^{2}-\hat{K}^{2}} \mathrm{~d} \hat{K}$

where we introduced the following notation:

$c_{ \pm}=c_{\mathrm{r}} \pm 1$.

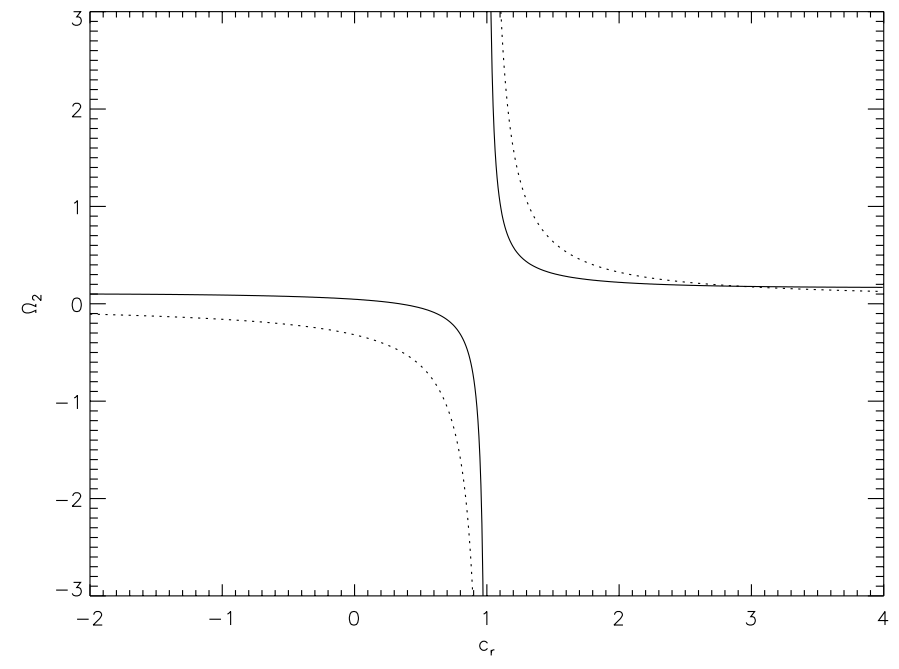

Fig. 3. Real (solid line) and imaginary (dashed line) parts of $\Omega_{2}$ for $K=2$.

It is noteworthy that the denominator in the integral becomes singular for $c_{-}=0\left(c_{\mathrm{r}}=1\right)$. This is the resonance case which we will defer to the forthcoming part of the paper. Now we consider the special case of $c_{+}=0\left(c_{\mathrm{r}}=-1\right)$. Evaluation of the above integral leads to the following expression:

$\Omega_{2}=\frac{1}{8 \pi^{3 / 2}} K(2-i \sqrt{\pi} K), \quad c_{\mathrm{r}}=-1$.

As the real (imaginary) part of $\Omega_{2}$ is positive (negative) we conclude that the rightwardly propagating sound waves are speeded up and attenuated by the wave noise which moves to the left with its speed $c_{\mathrm{r}}=-1$.

For $c_{\mathrm{r}} \neq \pm 1 \mathrm{Eq}$. (8) can be rewritten as follows:

$\Omega_{2}=\frac{1}{2 \pi^{2}} K \int_{-\infty}^{\infty} \frac{\left(c_{\mathrm{r}} \hat{K}-c_{-} K\right)^{2} \mathrm{e}^{-(\hat{K}-K)^{2}}}{c_{-} c_{+}(\hat{K}-K)\left(\hat{K}-\frac{c_{-}}{c_{+}} K\right)} \mathrm{d} \hat{K}$.

Hence

$$
\begin{aligned}
\Omega_{2}= & \frac{K}{2 \pi^{3 / 2}}\left[\frac{c_{\mathrm{r}}^{2}}{c_{\mathrm{r}}^{2}-1} K D\left(\frac{2}{c_{+}} K\right)\right] \\
& +i \frac{K^{2}}{4 \pi}\left[\frac{1}{c_{-}}+\left|\frac{c_{-}}{c_{+}}\right| \frac{1}{c_{+}} \exp \left(-4 K^{2} / c_{+}^{2}\right)\right] .
\end{aligned}
$$

The case $K=0$ has to be dealt with separately: fom Eq. (2) we find directly $\Omega=0$, which is also the limit of solutions in Eqs. (10)-(12) as $K \rightarrow 0$. This case corresponds to a "wave" of infinite wavelength which introduces no density variation in the medium and should be considered as part of the homogeneous background density.

Real and imaginary parts of $\Omega_{2}$ which follow from Eq. (12) are displayed in Fig. 1 versus the wavenumber $K$ and the random phase speed $c_{\mathrm{r}}$. As $\Re\left(\Omega_{2}\right)>0$ everywhere except in the region close to $c_{\mathrm{r}}=1$, we claim that the sound waves are speeded up by the wave noise there. Indeed, the top panel of Fig. 2 shows that for $c_{\mathrm{r}}=-2$ the real part of the frequency shift is positive for all values of (9) $K$. The imaginary part of the frequency shift is negative 
for all values of $K$. As a consequence of that we claim that for $c_{\mathrm{r}}=-2$ the sound waves are accelerated and attenuated for all $K$. The bottom panel of Fig. 2 illustrates that for $c_{\mathrm{r}}=2$ the real and imaginary parts of the frequency shifts are positive and the sound waves are accelerated and amplified by the wave noise.

At the place where the phase speed of the wave noise equals the sound wave speed a resonance occurs. Figure 3 shows this resonance for $K=2$. Note that the resonance is of the $1 / c_{\mathrm{r}}$-type; for $c_{\mathrm{r}}=1^{-}\left(c_{\mathrm{r}}=1^{+}\right)$the real and imaginary parts of the frequency shift are negative (positive) and the sound waves are decelerated and attenuated (accelerated and amplified) there. The wave deceleration and attenuation can be explained on physical grounds as for $c_{\mathrm{r}}=1^{-}$the sound wave interacts with slower propagating wave noise. This process is accompanied with an energy transfer from the sound wave into the wave noise, leading to the sound wave deceleration and attenuation. On the other hand, in the regime $c_{\mathrm{r}}=1^{+}$the wave noise moves quicker than the sound wave and the energy is transferred into the latter one. As a consequence of that the sound wave is amplified and speeded-up.

We draw the attention to the fact that, in the framework of our perturbative approach, the solutions we provide in Eqs. (10)-(12) and in Figs. 2-3, are consistent only if $\Omega_{2}$ remains $\mathcal{O}(1)$. In Fig. 2, this holds true only if $K<5$ and, in Fig. 3, only if $\left|c_{\mathrm{r}}-1\right|>0.1$.

\section{Summary}

In this paper we studied the propagation of plane sound waves in a medium with wave noise as a source of random mass density fluctuations. To the best of our knowledge the wave noise is introduced here for the first time in the random wave theory. The major result of our investigation is that these waves experience resonance when their phase speed $\omega / k$ equals the phase speed of the wave noise $c_{\mathrm{r}}$. For $c_{\mathrm{r}}>\omega / k$ the sound waves speed-up and become unstable as they propagate. This latter possibility is akin to the the case of a time-dependent random density field, which was discussed by Murawski et al. (2001a).

Acknowledgements. This work was financially supported by the State Committee for Scientific Research in Poland, KBN grant No. 2 PO3D 017 17, the Italian Research Council, and INTAS 97-31931. The authors express their thanks to an unknown referee for his/her comments on the earlier version of this paper.

\section{References}

Dziembowski, W. A. 2000, in Adv. Solar Res. at Eclipses from Ground and from Space, ed. J.-P. Zahn, \& M. Stavinschi (Kluwer, Amsterdam)

Gough, D. O. 1994, in Astrophysical Fluid Dynamics, ed. J.-P. Zahn, \& J. Zinn-Justin (Elsevier, Amsterdam)

Murawski, K. 2000a, A\&A, 360, 707

Murawski, K., Nocera, L., \& Mȩdrek, M. 2001a, A\&A, 376, 708

Murawski, K., Mędrek, M., \& Ostrowski, M. 2001b, in Proc. of the INTAS Workshop on MHD waves in Astrophysical Plasmas, ed. J. L. Ballester, \& B. Roberts

Nocera, L., Mędrek, M., \& Murawski, K. 2001, A\&A, 373, 301

Press, W. H., Teukolsky, S. A., Vetterling, W. T., \& Flannery, B. P. 1992, Numerical recipes in Fortran. The art of scientific computing (Cambridge University Press, Cambridge)

Sobczyk, K. 1985, Stochastic wave propagation (ElsevierPWN, Warsaw)

Stein, R., \& Nordlund, A. 2000, Sol. Phys., 192, 91

Swisdak, M., \& Zweibel, E. 1999, ApJ, 512, 442 